\title{
Fokus Maxim Gorki Theater Berlin
}

\author{
Ein kurzes Interview mit dem neuen Intendanten Armin Petras, \\ ergänzt durch Erläuterungen der dortigen Theaterpädagogin \\ Bärbel Jogschies
}

\author{
Bärbel Jogschies, Manfred Schewe
}

\section{Vorgeschichte}

Als Bärbel Jogschies im November 2006 an der Universität Cork über ihre Arbeit als Theaterpädagogin am Maxim Gorki Theater Berlin berichtete, entstand im Gespräch mit SCENARIO-Mitherausgeber Manfred Schewe die Idee eines Interviews mit Armin Petras (alias Dramatiker Fritz Kater), dem neuen Intendanten des Maxim Gorki Theaters. Nun ist dieser Mann ein engagierter Künstler, der auf vielen Hochzeiten gleichzeitig tanzt, ein Workoholic, der oft an mehreren Inszenierungen parallel arbeitet und nebenher auch noch Stücke schreibt. Armin Petras bat um die Zusendung von Fragen per E-Mail, er würde sie dann unterwegs beantworten. Unser gemeinsames Brainstorming mündete in 16 SCENARIO-Fragen. Die Antworten von Armin Petras (AP) kamen sofort zurück, waren allerdings so kurz und knapp, dass wir Erläuterungen für nötig hielten. So kam es zu der recht ungewöhnlichen Form dieses Beitrags. Entsprechende Initialien markieren die vertiefenden Nachfragen von Manfred Schewe (MS) und die darauf Bezug nehmenden Erläuterungen von Bärbel Jogschies (BJ). .

Der Artikel soll Interessierten einen Einblick in die deutsche Theaterpraxis und einige ihrer historischen Hintergründe gewähren. Über die theaterpädagogische Arbeit am Theater selbst wird in künftigen SCENARIO-Ausgaben genauer berichtet werden.

\section{Fragen an Armin Petras / Fritz Kater}

SCENARIO (1): Theater in Deutschland - hoch subventioniert und breit gestreut, traditionell Ausdruck bürgerlicher Selbstverständigung, Schlachtfeld des ideologischen Kampfes im Kalten Krieg und nun möglicherweise Opfer zunehmender Ökonomisierung, Nummer 1 auf der Streichliste für Einsparungen. Wie reagiert die Kunst? Wie reagiert das Gorki? Wie reagiert Armin Petras? Wie sehen Sie die Situation der Theater bzw. des Theaters in Deutschland. 
AP: Das Gorki und auch andere Theater reagieren durch Vernetzung untereinander sowie mit der Suche nach Sponsoring und Möglichkeiten, die Subventionskürzungen aufzufangen, z.B. durch

- Vereinfachung der Bühnenbilder,

- Grundräume für eine ganze Spielzeit,

- kleinere Produktionen.

(MS): Interessant wäre die Situation des deutschen Theaters im internationalen Vergleich, wobei wohl heraus käme, dass trotz der Einsparungen etc. die Situation immer noch eine privilegierte ist.

(BJ): Also, Deutschland hat eine über das ganze Land verteilte, hoch subventionierte Theaterlandschaft, die sich mit der Emanzipation des städtischen Bürgertums und höfischen Aufklärungsbestrebungen seit dem 18. Jahrhundert entwickelt hat. Theater ist fester Bestandteil öffentlicher kultureller Bildung und bürgerlich- demokratischen Selbstverständnisses. Neben Privattheatern und der freien Szene gibt es in Deutschland 150 öffentlich getragene Stadt- und Staatstheater und Landesbühnen und 130 Opernhäuser und Orchester, die jährlich 2 Milliarden Euro Finanzierung erhalten. Das sind ca. 0,2 \% der öffentlichen Ausgaben. Charakteristisch für Deutschland ist das mehrspartige Repertoiretheater mit festem Ensemble, das somit quasi der Lieferant einer kulturellen Grundversorgung einer Region ist. Natürlich wird dieser Luxus im Zuge zunehmender Ökonomisierung deutlich angefochten. In der Spielzeit 2004/05 gingen die öffentlichen Zuwendungen um 36 Millionen Euro zurück.Was für ganz Deutschland gilt, ist auch für Berlin ablesbar. Das Maxim Gorki Theater als kleinstes von 5 Staatstheatern mit eigenem Ensemble und einem Budget von 9 Mio Euro muss in der kommenden Spielzeit mit 180.000 Euro weniger auskommen.

SCENARIO (2): Theater in Berlin - Kampf der Kulturszene um Marktanteile und Publikumsgruppen, zwischen Überproduktion und Sparzwang. Das Motto in Berlin: „Wir sind arm aber sexy.“ (Bürgermeister Wowereit). Wie versteht sich das Gorki? Wo steht es zwischen Schaubühne, Deutschem Theater und Volksbühne?

AP: Das Gorki steht zwischen diesen Theatern und versteht sich als ein Theater für die Stadt Berlin.

(MS): Es wäre gut, mehr über das Profil der anderen Theater zu wissen, um dann das "Dazwischen"besser beurteilen zu können.

(BJ): Die Situation der Theater ist gleich und besonders. Die Stadt hat ca. 4 Mio. Einwohner. Es gibt 5 Staatstheater, 3 Opernhäuser, 2 Konzerthäuser und eine riesige Menge von Privattheatern und einer unüberschaubaren freien Szene. Aus dem Ensemble und dem Spielplan ergibt sich das künstlerische Profil eines Hauses. Ich nenne nur einige Theater, um Unterschiede und Überschneidungen deutlich zu machen: 
Das Deutsche Theater tritt das Erbe von Max Reinhardt an und war das künstlerisch am höchsten entwickelte Theater der DDR. Es steht für eine hohe ästhetische Form, klassische Stoffe, hochartifizielle Schauspiel- und Regiekunst. In der letzten Spielzeit widmete sich das Deutsche Theater explizit antiken Tragödien. Die Inszenierung der "Orestie" vom Oberspielleiter Michael Thalheimer war ein Beitrag zum Berliner Theatertreffen. Das absolute Gegenteil liefert die Volksbühne unter der Leitung des Stückezertrümmerers Frank Castorf. Dort setzte man sich in der letzten Spielzeit anhand von Stücken russischer Autoren kritisch mit medial vorgegebenen Wahrnehmungsrastern auseinander. Die Schaubühne am Lehniner Platz ist künstlerisch geprägt durch Regisseur Frank Ostermeier und die Choreografin Sascha Waltz. In Inszenierungen wie dem,Sommernachtstraum' sind die Grenzen der zwischen Schauspiel und Tanz nicht mehr sichtbar. Das Berliner Ensemble unter der Intendanz von Klaus Peymann stellt sich dem Brechtischen Erbe des politischen Theaters. Dass sich das Maxim Gorki Theater innerhalb dieser Landschaft nur schwer positionieren läßt, ist einerseits der Stadttheaterkonzeption geschuldet, die ganz klar erst einmal mit breitem Angebot auf den Markt geht um zu sehen, was beim Publikum ankommt. Zum Anderen braucht ein neues Ensemble Zeit, um sich zu formen und sein eigenes künstlerisches Profil zu entwickeln.

SCENARIO (3): Was ist gemeint mit der Stadttheaterkonzeption?

AP: Stadttheaterkonzeption steht für:

- Generationsüberschreitung;

- Überwindung der Diskrepanz zwischen Ost/ West;

- Behandelt aktuelle Themen und Fragestellungen, die die Gesellschaft betreffen;

- Moderne und aktuelle Spielweise.

(MS): Beispiele für äktuelle Themen und Fragestellungen, die die Gesellschaft betreffen", wären in diesem Kontext interessant.

(BJ): Zur Stadttheaterkonzeption hat Petras einen interessanten Artikel in einer Publikation des Deutschen Bühnenvereins geschrieben. ${ }^{1}$ In "Muss Theater sein?" beschäftigen sich verschiedene renommierte Theatermacher mit den ökonomischen und bevölkerungspolitischen Entwicklungen in Deutschland und ihren Auswirkungen auf das Theater. Da wird wieder einmal der „Tod des Theaters beschworen“, aber das ist, wie wir ja schon festgestellt haben, typisch deutsches Jammern auf sehr hohem Niveau. Armin Petras aber geht in seinem Artikel von der zunehmenden Verödung der speziell ostdeutschen Provinz aus. Die Industrien sind

\footnotetext{
${ }^{1}$ Deutscher Bühnenverein Bundesverband Deutscher Theater (Hrsg.) (2003): Muß Theater sein? Köln: Deutscher Bühnenverein (verantwortlich: Rolf Bolwin und Ulrich Khuon).
} 
zu Tode konkurriert. Die Landwirtschaft ist nur noch für ökologische Kleinbetriebe halbwegs wirtschaftlich. Die Menschen wandern dahin aus, wo es noch Arbeit gibt. Das Theater bekennt sich zu seiner Region und übernimmt die Aufgabe der Vereinigung der Hiergebliebenen, weiter lebend in der Illusion ihrer eigenen Legenden. Tribalisierung nennt er den Vorgang. „Tribalisierung von Theater bedeutet die inhaltliche, personelle und funktionelle Anbindung von Theaterstrukturen an eine Region... Die jeweilige mentale, kulturhistorische und soziale Situation ist genauestens zu untersuchen und für die Theatermacher, bei Strafe ihres Untergangs, unbedingt zu beachten." Diese Untersuchungen finden statt und finden im Maxim Gorki Theater ihren Niederschlag. Der „Prinz von Homburg“ von Heinrich von Kleist in der Inszenierung von Armin Petras wählt beispielsweise einen Zugriff und ein Zeichensystem, das jugendliches Publikum aus Berliner Randbezirken sofort und unmittelbar versteht. Homburg ist einer jener nach rechts tendierenden Bomberjackenjungs, der sich ins gute alte Preußentum der Kriegshelden und Kurfürsten und Prinzessinnen zurückträumt. Musik „Böhse Onkelz“ - „Bin ich nur glücklich, wenn es schmerzt?" Der Traum wird zum Albtraum und am Schluß steht der Held im Regen und kriegt keinen Applaus. Als Theaterpädagogin sehe ich die unmittelbare emotionale Wirkung auf ein Publikum von 15-18jährigen, die man sonst mit Theater und Kultur nur nervt. Hier wird eine Zielgruppe mit einer Inszenierung genauestens gespiegelt und abgeholt. In Fritz Katers Stück „Heaven“, das Armin Petras in der kommenden Spielzeit im Maxim Gorki Theater inszenieren wird, ist die Korrosion der ostdeutschen Landschaft Gegenstand des Stückes. Kater variiert das Tristanthema vor seiner Heimatstadt Wolfen, einem ehemaligen Zentrum der DDR-Chemieindustrie, heute Beispiel für Arbeitslosigkeit, Abwanderung, und Verwahrlosung einer ehemaligen Industriekultur. Das Muster Wolfen lässt sich auf zahllose ostdeutsche Städte übertragen (Bitterfeld, Schwedt, Schkopau) und es gilt auch für Randgebiete Berlins.

SCENARIO (4): Welchen Platz nehmen junge Zuschauer in dieser Konzeption ein?

AP: Junge Zuschauer sind uns wichtig, gleichsam junge Regisseure und Künstler. Das Gorki hat die jüngsten Regisseure der Stadt.

(MS): Wenn arm ßexyïst, ist das scheinbar das Gleiche mit "jung"? Warum dieser Fokus auf jung?

(BJ): Tja, da hast du den Finger auf einem wunden Punkt. Vielleicht zielt das darauf, dass der schon 43jährige Armin Petras sich selbst als Vater begreift und frühzeitig Platz für Nachwuchs schafft. Das ist eine deutliche Abgrenzung zu Petras Lieblingsfeuillitonfeind Claus Peymann, der als Prototyp der ewig jungen 68er Generation, weder ans Abtreten noch an Machtübergabe denkt. Petras hat mal Peymanns Theater das Rentnertheater genannt. In der kommenden Spielzeit wird das Thema Alter und das Thema Jugend wieder Schwerpunkt sein. 
SCENARIO (5): Theater in der Lehre - Pädagogik im Theater. Der erhobene Zeigefinger des Belehrungstheaters ist allen ein Graus. Man spürt die Absicht und ist verstimmt. Aber wenn man bedenkt, das Theater laut Müller das Laboratorium sozialer Erfahrung ist, das Lehrstücke von Brecht eine sehr moderne didaktische Konzeption von multiperspektivischem Lernen ist, da könnte es sehr wohl Durchdringungen der lernenden Systeme Theater und Schule bzw. Universität geben, nicht wahr?

AP: Uns ist die Zusammenarbeit mit den Theaterpädagogen aus dem Grunde wichtig, weil wir das Publikum zum kritischen Sehen und zu einer Auseinandersetzung und Hinterfragung erziehen wollen.

(MS): DieTheaterarbeit wird also sehrgeleitet von einer"gesellschaftskritischen Perspektive"; ähnlich wie an Schaubühne oder anders?

(BJ): Theatermacher verstehen sich in Deutschland sehr stark als Opposition zur spektakelheischenden Logik der Pressemedien und so als Teil eines gesamtgesellschaftlichen Selbstverständigungsprozesses. Das kritische Hinterfragen aktueller Vorgänge unter Bezugnahme auf historische Modelle gilt in nahezu allen deutschen Theatern als Auftrag. Dieser hohe politische Anspruch geht auf die Entstehung der deutschen Theaterkultur als Werkzeug der Aufklärung und bürgerlicher Emanzipation zurück. Er wurde im Westen durch die 68er Bewegung erneuert, die ganz neue politisch eingreifende Theaterformen kreierte. Im Osten konnte allein das Theater, im Gegensatz zu allen anderen Medien, geprägt durch die kritische Methode Brechts und eine hochentwickelte Kommunikation zwischen Schauspielern und Publikum, politische Botschaften an der Zensur vorbei übermitteln. Mit dieser politischen Relevanz rechtfertigen auch heute noch die Theatermacher ihre öffentlichen Bezüge. Die Apologie und Unterhaltung ist Sache der Privattheater. Ich habe vorhin schon Beispiele für diese Theaterarbeit genannt.

SCENARIO (6): In einem der letzten Hefte von Theater heute ging es um den Aspekt Vermittlung im professionellen Theater. ${ }^{2}$ Sehen Sie als Regisseur sich überhaupt in einer Vermittler-Rolle? Wenn ja, zwischen was oder wem vermitteln Sie - und in welcher Absicht?

AP: Jeder Regisseur ist in gewisser Weise ein Vermittler. Er vermittelt den Text des Autors über das Medium der Schauspieler. Die sind für den Regisseur enorm wichtig, denn je intensiver sie spielen, desto besser kann der Regisseur seine Intention vermitteln.

(MS): Gäbe es ein anschauliches Beispiel für diese Regie-Intention"?

(BJ): Beispiel für einen Schauspieler, der die Intention seines Regisseur bestens versteht und trägt, ist Robert Kuchenbuch, der spielt den Prinz Friedrich Arthur von Homburg. Endprobe. Die Vorhangordnung wird geprobt. Das Stück geht damit aus, dass der Brandenburger Jüngling sein Leben dem Führer/ Kurfürst übergibt. Wird er erschossen? Die Schlacht und der

\footnotetext{
${ }^{2}$ Vgl. Bericht über die Jahrestagung der Dramaturgischen Gesellschaft unter dem Titel „Kunst oder Bildung? Autonomie oder Arbeit in der Wissensgesellschaft?" In: Theater Heute 3 (2007), 4-9.
} 
Regen heben wieder an. Der Jüngling erwacht mit der Bierpulle in der Hand geht noch seinen Traumfiguren nach, die sich in die Vergangenheit zurückziehen und steht jetzt mit dem Rücken zum Publikum im Regen. So endet das Stück! Jetzt will es die Tradition, dass sich die Schauspieler verbeugen und das Publikum klatscht. Petras läßt alle Schauspieler außer Kuchenbuch vortreten und sich verbeugen. Petras sagt: „Ich find keinen Punkt, wo du dich verbeugen könntest!“ Kuchenbuch sagt: „Klar, dann bleib ich hier stehen" und so endet der Theaterabend. Die Schauspieler verbeugen sich bis auf den Brandenburger Nazi, der bleibt im Regen stehen! „Bin ich nur glücklich, wenn es schmerzt?“ Das Publikum klatscht und klatscht, ist irritiert, bemerkt nun die Absicht von Regisseur und Schauspieler und klatscht nochmal, extra für Kuchenbuch, der sich nicht umdreht und nicht verbeugt. Dann beginnt der Wettbewerb, wer länger durchhält, das Publikum oder der Schauspieler im Regen. In der Zeit wird gesprochen, diskutiert, man geht raus, kommt wieder rein... Der Nazi steht in Regen, der Schauspieler steht im Regen... bis der letzte Zuschauer geht und das kann manchmal eine Stunde dauern.

\section{SCENARIO.}

(7): Am Gorki-Theater haben Sie eine hervorragende theaterpädagogische Infrastruktur. Wie würden Sie - aus Ihrer Perspektive - kurz zusammenfassen, worin die Funktion der Theaterpädagogik an einem professionellen Theater besteht?

AP: Siehe Frage 5.

(MS): Ja, die Fragen 7 und 5 sind natürlich miteinander verknüpft.

(BJ): Hier antworte am besten ich: Die Theaterkunst, besonders unter so fruchtbaren Voraussetzungen wie in Deutschland, entwickelt ihre Zeichensysteme und Spielweisen rasant schnell weiter. Aber Theater realisiert sich nur im Kontakt mit dem Publikum, dem es eine gewisse Kennerschaft abverlangt. Brecht forderte die Entwicklung einer Zuschaukunst. Diese Arbeit leisten die Theaterpädagoginnen, die es mittlerweile an fast allen Theatern gibt. Sie öffnen durch Einführungen, Workshops, Jugendklubrabeit und Probenbesuche den Roten Vorhang und gewähren einen lebendigen Einblick in die Arbeitsprozesse des Theaters Der Zuschauer soll das Theater nicht nur konsumieren und dann nach seinem Geschmack bewerten, wie es ihm gefällt. Er soll koproduzieren. Er soll verstehen, was er sieht, sich seinen Teil dazu denken und Stellung nehmen. Er soll ernst genommen und am Prozess und Diskurs beteiligt werden. Im Maxim Gorki Theater Berlin besteht einer unserer Arbeitsschwerpunkte in der Gestaltung und Konzeption Inszenierungsbezogener Workshops für Schulklassen, die von den Berliner Lehrern rahmenplangerecht in den Unterricht einbezogen werden können. Der Unterricht findet also für einige Stunden im Theater statt und der Höhepunkt ist der Vorstellungsbesuch. Dabei ist Theater nicht nur Gegenstand sondern auch Methode der Workshops. Wir vermitteln Theater mit den Mitteln des Theaters. Viele Lehrer nutzen gern unsere Angebote auch als Anregung für ihre 
eigene Unterrichtsgestaltung und die Schüler lieben diese lebendige Abwechslung vom Schulalltag. Darüberhinaus bieten meine Kollegin Janka Panskus und ich spezielle Veranstaltungen für theaterinteressierte Studierende an. Die enge Nachbarschaft zur Humboldt-Universität legt eine solche Partnerschaft nahe. Grundsätzlich aber sind wir in der Lage, jeder Besuchergruppe eine maßgeschneiderte Vorbereitung auf ihren Theaterabend anzubieten.

SCENARIO (8): Unsere Zeitschrift SSCENARIO"beschäftigt sich schwerpunktmäßig mitAspekten der Vermittlung von Kultur, Literatur und Sprache durch Theater. Inwiefern setzen sich Ihre Stücke mit deutscher Kultur auseinander - Kultur hier natürlich in einem weiten Sinne verstanden?

AP: Alles, was in Deutschland passiert ist automatisch deutsche Kultur, insofern findet im Theater permanent eine Auseinandersetzung mit deutscher Kultur statt.Wir sind an der Geschichte des Landes interessiert und begeben uns in all unseren Vorhaben auf eine Spurensuche des Landes und der Menschen. Wir versuchen, Sozialisationsformen zu erforschen und dem auf den Grund zu gehen, was die Menschen dieses Landes ausmacht.

(MS): SSozialisationsformen erforschenmacht mich neugierig. Vielleicht hast Du ein konkretes Beispiel dafür, was hier gemeint sein könnte?

(BJ): Beispiel: "Heaven"von Kater:Die Übriggebliebenen von Wolfen, einstmals selbstbewußteIndustriearbeiter heute, Geradesozurechkommer zwischen ABM und Hartz 4, Nur Anders hat als Architekt den Absprung in die große weite Welt geschafft. Aber seine Freundin Simone hängt an ihrer sterbenden Heimat und bringt sich lieber um, als dass sie ihm folgen könnte. Pulsadern werden durchtrennt, Ehen scheitern, Herzen brechen. . . Anders beobachtet aus dem Flugzeug die neuen Völkerwanderungen von Süden nach Norden, von Osten nach Westen, aus der Armut in die Fülle, aus der Enge in die Weite, aus dem Hunger an die Fleischtöpfe, aus dem Überdruß in die Leere. Sehr spannend und sehr typisch für das Gorki Theater. Weitere Sozialisationsformen, die erforscht werden: das Leben der Alten im Altenheim, das Leben aller mit dem Klimawandel, das Leben der Jugend.

SCENARIO (9): Ist Fritz Kater ein deutscher Autor? Würden Sie Ihre Stücke als kulturspezifisch beschreiben?

AP: Ja, Fritz Kater ist ein deutsch-deutscher Autor.

(MS): Ließe sich dieser deutsch-deutsche Aspekt weiter entfalten?

(BJ): Klar, das spannende an Petras' Biografie ist, dass er als Kind mit seinen Eltern aus dem Westen in den Osten kam und dass er als junger Mann wieder in den Westen ging. So kann er aufgrund unmittelbarer Lebenserfahrung in beiden Systemen etwas über ihre Verwandlungen berichten. Fritz Kater ist übrigens im Osten geboren und 1987 in den Westen abgehauen, wo er sich in Bayern mit einfachen Jobs durchschlug bevor er 1990 nach Berlin zurückkam und zu schreiben begann. Die ehemalige Teilung Deutschlands führt nach wie vor zu Verwerfungen und 
Spannungen, die gerade für Dramatiker ein willkommenes Futter für ihre Stücke darstellen. Westdeutsch ist nicht gleich ostdeutsch und bedarf der Übersetzung.

SCENARIO (10): Deutschstudierende an ausländischen Universitäten lesen und inzenieren gelegentlich auch Stücke von deutschsprachigen Autoren, um auf diese Weise etwas über Deutschland zu lernen. Welches Ihrer Stücke würden Sie den Studierenden speziell empfehlen und warum gerade dieses?

AP: Ich würde Studierenden das Stück von Thomas Lawinky und Armin Petras, „mala zementbaum“, empfehlen. ${ }^{3}$ Hier gibt es drei Zeitebenen, die etwas über deutsche Geschichte beschreiben. Außerdem entlarvt es die Unterstellung, politisches Leben sei mit einem schwarz/weiß-Schema zu bewerten. Das stimmt nicht, dieser Verdacht geht am realen Leben vorbei.

SCENARIO (11): Sie setzen sich als Regisseur intensiver mit Heinrich von Kleist auseinander. Was fasziniert Sie an Kleist?

AP: Kleist ist der zerrissenste Autor, den ich kenne. Dies meine ich auf sein Leben und den Anspruch, den er an sich selber stellt, bezogen. Er litt an typisch deutschen Leiden, Problemen und Zuständen, an denen er letztendlich auch zugrunde ging. Zudem ist er der sprachmächtigste deutsche Dichter.

(MS): Kleist ist ja auch in der theaterpädagogischen Arbeit des Maxim Gorki Theaters ein besonderer Fokus, oder?

(BJ): Lieber die Theaterpädagogik zu Kleist in einem Extra-Artikel, hier als Kommentar vielleicht nur noch so viel: Kleist ist Schwerpunktautor in unserem Theater. Seine Biografie ist mit preußischer Geschichte und Berlin verbunden. Bisher spielen wir "Das Käthchen von Heilbronn" und "Prinz Friedrich von Homburg". In der kommenden Spielzeit wird unser Hausregisseur Jan Bosse den "Amphitryon" inszenieren. Jan Bosse ist derzeit einer der erfolgreichsten deutschen Regisseure. Seine "WertherInszenierung" war gerade zum Berliner Theatertreffen eingeladen. Wir dürfen also sehr gespannt sein.

SCENARIO (12): Inwiefern haben - neben Kleist - noch andere Autoren bzw. Autorinnen sie beeinflusst?

AP: Neben Kleist haben mich die Autoren Einar Schleef, Fassbinder, Georg Büchner, Schiller, Shakespeare und Grabbe beeinflusst.

SCENARIO (13): Ihre Stücke sind für Deutschstudierende im Ausland eine besondere Herausforderung aufgrund der fehlenden Punkte, Kommas etc. Könnten Sie kurz etwas zu diesem ungewöhnlichen Umgang mit der deutschen Sprache sagen? = Fritz Kater schreibt Alltagssprache ohne Punkt und Komma. Kommen die Texte so aus den Fingern in die Tastatur gelaufen? Sind das Schreibflashs? Müller meinte, er müsse viel trinken, damit seine Texte die Qualität seiner Träume erreichen könnten? Wie schreibt Kater?

\footnotetext{
${ }^{3}$ Ein Stückabdruck findet sich in Theater Heute 2 (2007), 53-59.
} 
AP: Da der dramatische Text in der Regel NUR gehört wird und nicht gelesen, ist die Interpunktion zweitrangig. Die Weise, die Kater schreibt, bietet Schauspielern und Regisseur die Option, die Sinne der Sprache selber zusammen zu setzen und ist entfernt von einer vorgegebenen Interpretation.

(MS): Einige Autoren geben sehr genaue Bühnenanweisungen, bestehen auf Texttreue, andere, so anscheinend auch Armin Petras, sind da wesentlich liberaler.

(BJ): Petras ist ein Regisseur, der nach eingehender genauer Beschäftigung mit dem Text sehr weit in das Stück eingreift und sogar die Geschichte verändert. Es kommt nur auf die Bühne, was der zu erzählenden heutigen Geschichte nutzt. Da kommt er klar aus der Castorf-Ecke: „Ein Text ist ein Material, das benutzt wird." Bei jedem Stück, das am Gorki gespielt wird, stellt die Strichfassung eine Bearbeitung im Sinne der gefundenen Lesart dar. Diese Fassungen müssen durch lebende Autoren autorisiert werden. Das gilt auch für Fritz Kater, der wird als Autor auch nicht besser behandelt.

SCENARIO (14): Schreiben und Inszenieren - Sie verfassen Texte und machen diese auch lebendig, indem sie diese oft selber inszenieren. Worin bestehen für Sie die Unterschiede bzw. auch Gemeinsamkeiten zwischen diesen Tätigkeiten? Wie ist das Verhältnis von Autor Fritz Kater und Regisseur Armin Petras?

AP: Mein Verhältnis zu Kater ist sehr gut, jeder macht seinen Job und lässt den anderen seinen tun.

(MS): Eine humorvolle Antwort.

(BJ): Das ist gar kein Witz, sondern ganz ernst gemeint als klare Trennung der Funktionen und Aufgaben! Der Regisseur Armin Petras zum Beispiel taucht als Koautor von "Mala Zementbaum" auf und auch als Bearbeiter von Filmdrehbüchern für die Bühne, wie zum Beispiel in "Gegen die Wand". Fritz Kater schreibt eigene Stücke. Angeblich ist er 3 Jahre jünger als Petras und arbeitet eigentlich in einer Werbefirma in Berlin Moabit. Er hat drei Kinder und taucht nur selten im Theater auf.

SCENARIO (15): Sie unterrichten auch im Studiengang Szenisches Schreiben. Kann man es lernen, gute Stücke zu schreiben? Und worauf kommt es beim Inszenieren besonders an? Hätten Sie zweckdienliche Hinweise für theaterbegeisterte junge Studierende?

AP: Mit Sicherheit kann man Dinge lernen, die einen beim Stückeschreiben hilfreich sein können. Natürlich muss man ein grundlegendes Talent mitbringen und es ist auch ein Talent, das Angebot, was der Lehrende macht, anzuwenden und für sich zu nutzen. Die wichtigste Haltung, die man beim Inszenieren haben sollte ist eine eigene Weltsicht auf die Dinge und eine klares Anliegen, das man an das Stück stellt.

SCENARIO(16): Wie ist die Bilanz nach einem Jahr Gorki? Es geht die Legende, Sie würden das Experiment entgegen der 5jährigen Vertragslaufzeit eines Intendanten auch vorzeitig abbrechen, wenn es nicht zufrieden stellend 
verläuft. Was war gut? Was nicht? Was sind die neuen Vorhaben in der nächsten Spielzeit?

AP: Die Bilanz der letzten Spielzeit kann man am besten anhand der Zahlen beschreiben. Die entbehren jeglichen Kommentars:

- 269 Vorstellungen

- 37 Gastspiele

und eine komplett erschöpfte Belegschaft. Für mich waren die positivsten Erfahrungen das Zusammentreffen mit neuen Menschen, unerfreulich waren einzelne halbherzige Inszenierungen.Die Erwartungen an die kommende Spielzeit sind hoch, wie wir sie angehen läßt sich unserem Spielzeitheft entnehmen.

(BJ): Ich würde diese Bilanz gerne ergänzen: ${ }^{4}$

- 44 Premieren (normal für ein Theater mit 2 Bühnen wären 25), davon viele als Koproduktionen mit anderen Stadttheatern oder Festivals;

- Etliche Preise für Stücke (Mala Zementbaum) und Inszenierungen;

- 150 theaterpädagogische Veranstaltungen mit ca 4000 Teilnehmern.

Der Einstieg des neuen Ensembles in die Berliner Theaterszene scheint geschafft und vom Publikum durch 5000 mehr verkaufte Karten bestätigt zu sein. Für die kommende Spielzeit gibt es nun ein Repertoire von 12 sehr guten Inszenierungen und 15 weitere Premieren sind geplant. Das Maxim Gorki Theater Berlin empfiehlt sich als Kulturtip für alle, die an sehr innovativem deutschsprachigen Theater interessiert sind. Und wer eine Einführung, Hintergrundwissen oder Übersetzungshilfe braucht, der ist bei den Theaterpädagoginnen Bärbel Jogschies und Janka Panskus herzlich willkommen: .

\footnotetext{
4 Siehe auch Christine Wahl: Die Selbstverausgabungsoffensive. Armin Petras bricht am Berliner Maxim Gorki Theater alle Premierenrekorde - und entwertet mit allzu viel Halbgarem eigene Highlights. In: Theater Heute 8/9 (2007), 50-52.
} 